\title{
Realistic Electric Field Mapping of Anisotropic Muscle During Electrical Stimulation Using a Combination of Water Diffusion Tensor and Electrical Conductivity
}

\author{
Bup Kyung Choi ${ }^{1}$, Tong In $\mathrm{Oh}^{2}$, Saurav ZK Sajib², Jin Woong Kim³ ${ }^{3}$ Hyung Joong $\mathrm{Kim}^{2}$, Oh In Kwon ${ }^{4}$, Eung Je Woo ${ }^{2}$ \\ ${ }^{1}$ Department of Biomedical Engineering, Kyung Hee University, Yongin, Korea \\ ${ }^{2}$ Impedance Imaging Research Center, Kyung Hee University, Seoul, Korea \\ ${ }^{3}$ Department of Radiology, Chonnam National University Medical School, Gwangju, Korea \\ ${ }^{4}$ Department of Mathematics, Konkuk University, Seoul, Korea
}

Purpose: To realistically map the electric fields of biological tissues using a diffusion tensor magnetic resonance electrical impedance tomography (DT-MREIT) method to estimate tissue response during electrical stimulation.

Methods: Imaging experiments were performed using chunks of bovine muscle. Two silver wire electrodes were positioned inside the muscle tissue for electrical stimulation. Electric pulses were applied with a $100-\mathrm{V}$ amplitude and $100-\mu$ s width using a voltage stimulator. During electrical stimulation, we collected DT-MREIT data from a 3T magnetic resonance imaging scanner. We adopted the projected current density method to calculate the electric field. Based on the relation between the water diffusion tensor and the conductivity tensor, we computed the position-dependent scale factor using the measured magnetic flux density data. Then, a final conductivity tensor map was reconstructed using the multiplication of the water diffusion tensor and the scale factor.

Results: The current density images from DT-MREIT data represent the internal current flows that exist not only in the electrodes but also in surrounding regions. The reconstructed electric filed map from our anisotropic conductivity tensor with the projected current density shows coverage that is more than 2 times as wide, and higher signals in both the electrodes and surrounding tissues, than the previous isotropic method owing to the consideration of tissue anisotropy.

Conclusions: An electric field map obtained by an anisotropic reconstruction method showed different patterns from the results of the previous isotropic reconstruction method. Since accurate electric field mapping is important to correctly estimate the coverage of the electrical treatment, future studies should include more rigorous validations of the new method through in vivo and in situ experiments.

Keywords: Electric Field; Electrical Stimulation; Anisotropy; Magnetic Resonance Electrical Impedance Tomography; Diffusion Tensor Imaging

- Fund/Grant Support: This work was supported by the National Research Foundation of Korea (NRF) grant funded by the Korea government (MEST and MSIP) (No. 2015R1D1A1A09058104, 2016R1A2B4014534, 2017R1A2A1A05001330).

- Conflict of Interest: No potential conflict of interest relevant to this article was reported.

\section{- HIGHLIGHTS}

- Precise consideration of tissue conditions such as tissue anisotropy is important when determining and estimating the therapeutic effects of electrical stimulation.

- Electric field map by an anisotropic reconstruction method showed higher and wider coverage between the stimulating electrodes responding to the electric pulses.

Corresponding author: Hyung Joong Kim (iD http://orcid.org/0000-0001-7591-9079 Impedance Imaging Research Center, Kyung Hee University, 23 Kyungheedae-ro, Dongdaemun-gu, Seoul 02447, Korea

E-mail: bmekim@khu.ac.kr / Tel: +82-2-958-2895 / Fax: +82-2-958-2889

Submitted: March 15, 2017 / Accepted after revision: April 7, 2017
This is an Open Access article distributed under the terms of the Creative Commons Attribution Non-Commercial License (http://creativecommons.org/licenses/by-nc/4.0/) which permits unrestricted non-commercial use, distribution, and reproduction in any medium, provided the original work is properly cited. 


\section{INTRODUCTION}

Noninvasive measurement of tissue responses when performing electrical stimulation on a patient is indispensible in ensuring the effectiveness of clinical applications [1]. Voltage-controlled electrical stimulation such as electroporation applies electric pulses with high intensity to enhance cell membrane permeability $[2,3]$. By controlling the membrane permeability, electroporation is highly efficient for the introduction of foreign genes into cells [4]. Despite its remarkable effects in cancer therapy, tissue response is still unclear owing to limitations in imaging capabilities. The development of electric field mapping methods for electrical stimulation is, therefore, essential for its applications in the clinical environment.

Magnetic resonance (MR)-based tissue property mapping is an emerging technique that utilizes MR scanner to obtain noninvasive information concerning electrical tissue properties such as conductivity and permittivity [5-8]. Such electromagnetic tissue properties provide alternative information on tissue structure and function, and can serve as a good complement to the information provided by traditional magnetic resonance imaging (MRI) methods [9-11]. The recent magnetic resonance electrical impedance tomography (MREIT) method enables the highly resolved imaging of electromagnetic tissue properties such as current density and conductivity by externally injected current [8,10,12]. Recently, Kranjc et al. [3] applied the MREIT method to electroporation, which is a typical voltage-controlled electrical stimulation, to map the electric field in biological tissues. From the measured current density and conductivity, the researchers imaged the electric field by simply applying Ampere's law and Ohm's law. However, there was no consideration for actual tissue conditions such as anisotropy.

Since biological tissues are composed of numerous components with or without anisotropic properties, the effects of tissue anisotropy should be considered in actual situations [13]. Recently, Kwon et al. [14] reported a novel MREIT method to map anisotropic conductivity by adopting a conductivity tensor model inferred from a water diffusion tensor. Their diffusion tensor MREIT (DT-MREIT) method images the directional information of water molecules from diffusion tensor imaging (DTI) and magnitude information from MREIT imaging. Finally, the researchers calculated a scale factor, which combines the water diffusion tensor with the local distribution of the current density measured from the z-component of the magnetic flux density. From numerical simulations and tissue phantom imaging experiments [15-17], the DT-MREIT images well demonstrated the enhanced signal intensity in strong anisotropic tissues.

The purpose of this study is to experimentally show the electric field of biological tissues during voltage-controlled electrical stimulation. Applying the DT-MREIT method to bovine muscle tissue with strong anisotropy, we imaged the electric field based on the current density and anisotropic conductivity of the muscle tissue. For comparison, we mapped the electric fields using both isotropic and anisotropic reconstruction methods. We measured the values of the electric field components for a quantitative analysis.

\section{MATERIALS AND METHODS}

\section{Setup for Imaging Experiment}

Five chunks of bovine muscle were used for the DT-MREIT imaging experiments (Fig. 1A). The bovine muscle was purchased from a local butcher. Each chunk arrived at our laboratory on the morning of the experiment, and the elapsed times from butchery were almost the same for all cases. The muscle was cut immediately into $15 \times 8 \times 8-\mathrm{cm}^{3}$ blocks for the imaging experiment at a room temperature of $20^{\circ} \mathrm{C}$. To apply electric pulses, two stimulating electrodes with a $30-\mathrm{mm}$ distance were inserted into the muscle tissue at a depth of $50 \mathrm{~mm}$ (Fig. 1B). The electrodes were insulated by a heat-shrink tube except for 2 $\mathrm{mm}$ at the tips. For electrical stimulation, 128 electric pulses were applied through the electrodes with a $100-\mathrm{V}$ amplitude and $100-\mu$ s width using a voltage stimulator (BIOPAC Systems Inc., Goleta, CA, USA) (Fig. 1C). The electric pulses from the voltage amplifier operated in synchronization with MR trigger signal (Fig. 1D). After muscle tissue preparation, the imaging object was located inside the bore of a 3T MRI system (Achieva TX, Philips, Amsterdam, the Netherlands) (Fig. 1A).

\section{DT-MREIT Imaging}

For DT-MREIT data acquisition, we first used a single-shotspin-echo echo planer imaging MR pulse sequence (repetition time $/$ echo time $=3,500 / 70 \mathrm{msec}$ ) to collect diffusion tensor data with $b$ values of $500 \mathrm{sec} / \mathrm{mm}^{2}$. In addition, reference MR data without a diffusion gradient were obtained to calculate the diffusion tensor. Then, we applied a multigradient-echo MR pulse sequence to acquire the induced magnetic flux density $\left(B_{z}\right)$ data during the electrical stimulation. The imaging parameters were as follows: TR/TE $=200 / 1.6 \mathrm{msec}$, ES (echo spacing) $=2.3 \mathrm{msec}$, 

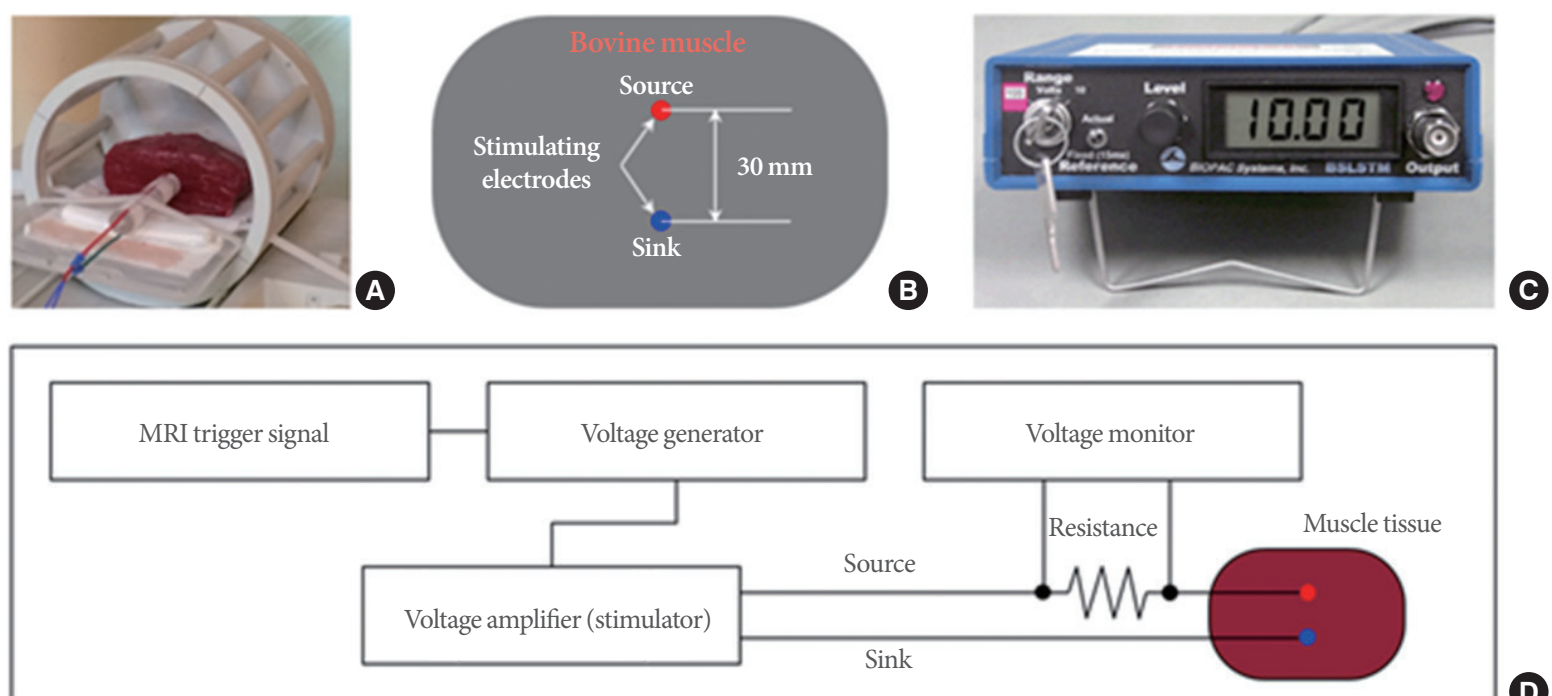

Fig. 1. Experimental setup for muscle tissue imaging. (A) Location of muscle tissue inside radiofrequency coil, (B) electrode configuration, (C) voltage stimulator, and (D) diagram of measuring voltage-controlled electrical stimulation. MRI, magnetic resonance imaging.

NE (number of echoes) $=32$, FOV (field-of-view) $=200 \times 200$ $\mathrm{mm}^{2}$, imaging matrix $=128 \times 128$, and imaging time $=51.2 \mathrm{sec}$. We collected the raw data of the DT-MREIT from the MR spectrometer after data acquisition. Using the raw data, we applied a previous simpler method ( $J$-substitution method) to the isotropic electric field and projected current density method, combining this with DTI information, for the anisotropic electric field.

\section{Electric Field Reconstruction}

To map the electric field, we first calculated the current density information of the muscle tissue. Since we could acquire only one component $\left(B_{z}\right)$ of the magnetic flux density $\mathrm{B}=\left(B_{x}, B_{y}, B_{z}\right)$ from the MREIT data, we applied the projected current density method for recovering 3-dimensional current density information from the measured $B_{z}$ data $[14,18]$ :

$$
\mathbf{J}^{P}=\mathbf{J}^{0}+\frac{1}{\mu_{0}}\left(\frac{\partial\left(B_{z}-B_{z}^{0}\right)}{\partial y},-\frac{\partial\left(B_{z}-B_{z}^{0}\right)}{\partial x}, 0\right)
$$

where $\mathrm{J}^{0}$ is the current density calculated from a 3-dimensional model with uniform conductivity subject to the same boundary conditions as used in the imaging experiments. $B_{z}{ }^{0}$ is the $\mathrm{z}$-component of $\mathrm{B}$ corresponding to the calculated current density $J^{0}$.

In this step, we assumed a linear relationship between the conductivity tensor and the water diffusion tensor to discriminate tissue anisotropy [14]. Specifically, the directional proper- ties of both water molecules and ions have the same conditions because they are in the same structural environment. Based on this relationship, a conductivity tensor (C) can be defined as a scalar multiple of the water diffusion tensor (D):

$$
\mathrm{C}=\eta \mathrm{D}
$$

where $\eta$ is the scalar factor. Using the diffusion tensor D obtained from a DTI scan and the recovered projected current density $J^{\mathrm{P}}$ from an MREIT scan, we can reconstruct the position-dependent scale factor $\eta$ as

$$
\eta=-\frac{\mathbf{J}^{P} \cdot \nabla u^{0}}{\mathbf{D} \nabla u^{0} \cdot \nabla u^{0}}
$$

where $u^{0}$ is the calculated voltage from the model with uniform conductivity distribution. The conductivity tensor C, then, is computed with this relationship. Finally, we obtained the conductivity tensor as a $3 \times 3$ matrix, and computed the electric field by multiplying the inverse of the conductivity tensor by the current density vector.

\section{RESULTS}

\section{DT-MREIT Images}

Fig. 2 shows typical results of DT-MREIT imaging during electrical stimulation. The MR magnitude image in Fig. 2A indicates the anatomical structure of the muscle tissue and the location of the stimulating electrodes. The color-coded fractional anisotropy map (Fig. 2B), which was obtained from the diffu- 



Fig. 2. Typical results of diffusion tensor magnetic resonance electrical impedance tomography imaging during electrical stimulation. (A) Magnetic resonance (MR) magnitude image of bovine muscle tissue, (B) color-coded fractional anisotropy (cFA) map, (C) measured magnetic flux density, and (D) projected current density distribution. L to R, left to right; A to P, anterior to posterior, S to I, superior to inferior.
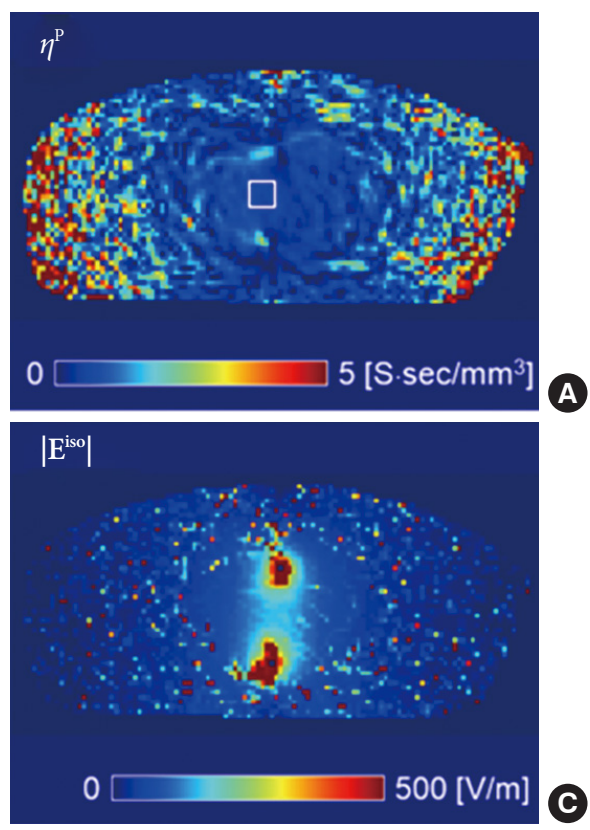
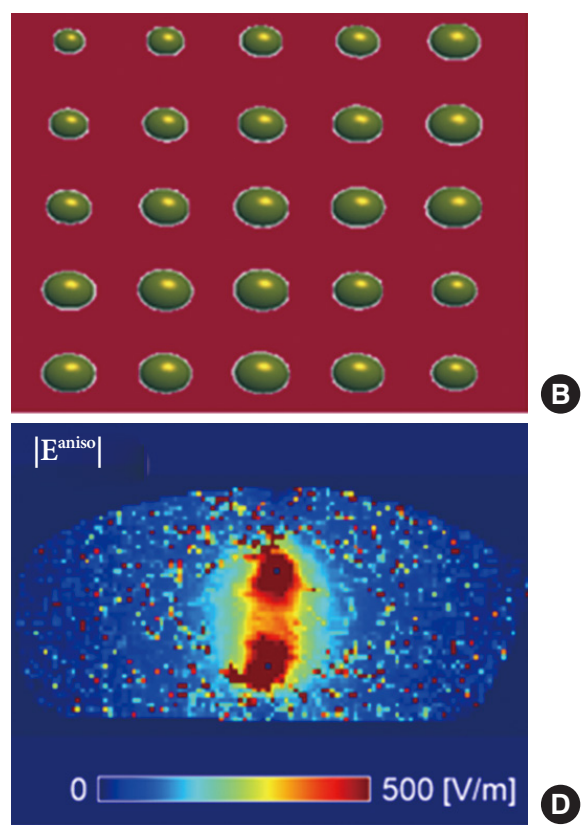

Fig. 3. Reconstruction of electric field map at muscle tissue. (A) Position-dependent scale factor map, (B) conductivity tensor information in region of interest, $(\mathrm{C})$ electric field map by previous isotropic conductivity reconstruction method, and (D) electric field map by anisotropic conductivity reconstruction method using diffusion tensor magnetic resonance electrical impedance tomography.

sion tensor image, shows the directional information of the muscle tissue in 3 directions. The map provides only informa- tion on ion mobility, without any information on the concentration of ions. The measured magnetic flux density $\left(B_{z}\right)$ and 
Table 1. Electric field (E) values of bovine muscles $(n=5)$ for isotropic and anisotropic reconstruction methods

\begin{tabular}{lcccc}
\hline Electric field & $\left|\mathrm{E}_{x}\right|$ & $\left|\mathrm{E}_{y}\right|$ & $\left|\mathrm{E}_{z}\right|$ & $|\mathrm{E}|$ \\
\hline Isotropic & $23.9 \pm 18.0$ & $155.1 \pm 9.9$ & $0.7 \pm 0.5$ & $158.1 \pm 8.7$ \\
Anisotropic & $51.9 \pm 37.2$ & $362.7 \pm 22.5$ & $28.3 \pm 7.9$ & $369.5 \pm 21.0$ \\
\hline
\end{tabular}

Values are presented as mean \pm standard deviation.

$\mathrm{E}_{x}$, directional information from left to right; $\mathrm{E}_{y}$, superior to inferior; $\mathrm{E}_{z}$, anterior to posterior.

projected current density $\left(\mathrm{J}^{\mathrm{p}}\right)$ images (Fig. 2C, D) provide prior knowledge for mapping the electric field when the therapeutic pulses are injected. The current density image indicates the internal current flows that exist not only at the electrodes but also in the surrounding regions.

\section{Electric Field Map of Muscle Tissues}

Fig. 3 shows the results of the electric field map for the muscle tissue. The position-dependent scale factor $\eta$ (Fig. 3A) was obtained by incorporating the measured diffusion tensor with the recovered projected current density. In this step, we implemented the anisotropy of the muscle tissue using the method that is mentioned in the methods section [14]. The elliptical conductivity tensor in Fig. 3B, which was obtained from the white rectangle in Fig. 3A, provides information on the intensity and direction of the tensor in the anisotropic muscle tissue. After recovering the conductivity tensor using the relationship in equation 2, we mapped the electric field of the muscle tissue. The electric field maps were reconstructed from the equivalent isotropic conductivity distribution (Fig. 3C) using the previous method and the anisotropic conductivity tensor (Fig. 3D) using the projected current density method. Comparing the two methods, the anisotropic reconstruction method had wider coverage and enhanced signals in the electrodes and surrounding tissues owing to the consideration of tissue property.

\section{Quantification}

Table 1 shows a comparison of electric field strength for the isotropic and anisotropic reconstruction methods. We measured the electric field values of the muscle tissue from a region of interest (ROI) of a $5 \times 5$-pixel size. The ROI was positioned between the 2 electrodes, and is marked in Fig. 3A. The signal intensity of the anisotropic method was more than 2 times higher than the results of the isotropy for the 3-directional and absolute electric fields. Since all experimental conditions were the same, the difference between the 2 methods originated from the degree of tissue anisotropy.

\section{DISCUSSION}

In the treatment of urinary disorders, electrical stimulation is considered to be a useful therapy for an irritable bladder. In addition, electrical stimulation can be used as an effective treatment to overcome the limitations of conservative treatment and anti-muscarinic drug therapy [19]. Electrical stimulations are widely used as therapeutic techniques that are closely related to the electric fields inside the human body $[2,3,20,21]$. Since the electric field is affected by the injected currents, voltages, and electrical conductivities of biological tissue, a map of voltage, current density, and magnetic flux density can provide meaningful information to monitor and estimate tissue responses [911]. Most biological tissues exhibit unique electromagnetic properties with a degree of anisotropic characteristic [12-17]. If we directly apply electric field estimation using an equivalent isotropic conductivity distribution, it could provide incorrect information about tissue conditions. When we mapped the tissue anisotropy, there was no imaging modality that could provide information on both the movement and concentration of existing ions.

Recent advances in biomedical engineering provide an opportunity to overcome technical limitations in mapping electromagnetic tissue properties with electrical stimulation [3, 19,20]. The DT-MREIT method successfully produced quantitative maps of anisotropic conductivity tensor distribution by combining the directional water mobility information from DTI and the ion concentration information from MREIT. In this study, we introduced a recent DT-MREIT method to better apply it to a real situation. The current density distribution of muscle tissues can provide intuitive information on the current pathway, which reflects the directional information by tissue type. The signal intensity of the current density is proportional to the magnetic flux density, which can be measured by MREIT [14-17]. The reconstructed conductivity tensor image can provide information on tissue response related to movement in the cellular environment and the average ion concentration in each 
voxel. DT-MREIT has advantages that can complement the limitations of existing MR-based tissue properties such as MREIT and magnetic resonance electric properties tomography. For more active translational research and applications in high-field MR systems, the signal-to-noise ratio must be improved, and the imaging time must be reduced, together with high-order shimming for field homogeneity, a precise phase unwrapping technique, and susceptibility artifact correction.

The electric field map obtained by the anisotropic reconstruction method showed different patterns from the result by the previous equivalent isotropic reconstruction method. Specifically, current density depends on the actual pathways of current flow inside the tissue, and shows their values when current flows are parallel to the fiber direction [22-24]. A contrast that is more than two times higher and wider coverage between the stimulating electrodes responding to the electric pulses indicates tissue conditions such as the degree of anisotropy. Our results suggest that the precise consideration of tissue conditions such as tissue anisotropy is important when determining and estimating the therapeutic effects of electrical stimulation. Though additional research related with this method should continue, electric field mapping using the anisotropic reconstruction method can be helpful for quantitative assessments of treatment outcomes after electrical stimulation therapy for diseases such as overactive bladder or chronic prostatitis in clinical practice. Moreover, it is necessary to establish therapeutic protocols for treatment guidelines, patient selection criteria, and effective therapy for relapsed patients.

In this study, we experimentally mapped the electric field of muscle tissue using a DT-MREIT method. Using the relationship between the diffusion tensor and conductivity tensor, we can reconstruct a realistic electric field map of the muscle tissue by enhancing the anisotropic tissue property. Further rigorous validations were performed. We expect that DT-MREIT will provide prior knowledge on tissue conditions to be used for in vivo human therapy.

\section{REFERENCES}

1. Yarmush ML, Golberg A, Serša G, Kotnik T, Miklavčič D. Electroporation-based technologies for medicine: principles, applications, and challenges. Annu Rev Biomed Eng 2014;16:295-320.

2. Miklavcic D, Beravs K, Semrov D, Cemazar M, Demsar F, Sersa G. The importance of electric field distribution for effective in vivo electroporation of tissues. Biophys J 1998;74:2152-8.
3. Kranjc M, Markelc B, Bajd F, Čemažar M, Serša I, Blagus T, et al. In situ monitoring of electric field distribution in mouse tumor during electroporation. Radiology 2015;274:115-23.

4. Neumann E, Schaefer-Ridder M, Wang Y, Hofschneider PH. Gene transfer into mouse lyoma cells by electroporation in high electric fields. EMBO J 1982;1:841-5.

5. Katscher U, Voigt T, Findeklee C, Vernickel P, Nehrke K, Dössel O. Determination of electric conductivity and local SAR via B1 mapping. IEEE Trans Med Imaging 2009;28:1365-74.

6. Seo JK, Kim MO, Lee J, Choi N, Woo EJ, Kim HJ, et al. Error analysis of nonconstant admittivity for MR-based electric property imaging. IEEE Trans Med Imaging 2012;31:430-7.

7. Voigt T, Katscher U, Doessel O. Quantitative conductivity and permittivity imaging of the human brain using electric properties tomography. Magn Reson Med 2011;66:456-66.

8. Kim HJ, Jeong WC, Sajib SZ, Kim MO, Kwon OI, Je Woo E, et al. Simultaneous imaging of dual-frequency electrical conductivity using a combination of MREIT and MREPT. Magn Reson Med 2014;71:200-8.

9. Haemmerich D, Staelin ST, Tsai JZ, Tungjitkusolmun S, Mahvi DM, Webster JG. In vivo electrical conductivity of hepatic tumours. Physiol Meas 2003;24:251-60.

10. Oh TI, Jeong WC, McEwan A, Park HM, Kim HJ, Kwon OI, et al. Feasibility of magnetic resonance electrical impedance tomography (MREIT) conductivity imaging to evaluate brain abscess lesion: in vivo canine model. J Magn Reson Imaging 2013;38:189-97.

11. Lee SK, Bulumulla S, Wiesinger F, Sacolick L, Sun W, Hancu I. Tissue electrical property mapping from zero echo-time magnetic resonance imaging. IEEE Trans Med Imaging 2015;34:541-50.

12. Seo JK, Woo EJ. Electrical tissue property imaging at low frequency using MREIT. IEEE Trans Biomed Eng 2014;61:1390-9.

13. Tuch DS, Wedeen VJ, Dale AM, George JS, Belliveau JW. Conductivity tensor mapping of the human brain using diffusion tensor MRI. Proc Natl Acad Sci U S A 2001;98:11697-701.

14. Kwon OI, Jeong WC, Sajib SZ, Kim HJ, Woo EJ. Anisotropic conductivity tensor imaging in MREIT using directional diffusion rate of water molecules. Phys Med Biol 2014;59:2955-74.

15. Kwon OI, Sajib SZ, Sersa I, Oh TI, Jeong WC, Kim HJ, et al. Current density imaging during transcranial direct current stimulation using DT-MRI and MREIT: algorithm development and numerical simulations. IEEE Trans Biomed Eng 2016;63:168-75.

16. Sajib SZ, Jeong WC, Kyung EJ, Kim HB, Oh TI, Kim HJ, et al. Experimental evaluation of electrical conductivity imaging of anisotropic brain tissues using a combination of diffusion tensor imaging and magnetic resonance electrical impedance tomography. AIP 
Adv 2016;6:065109.

17. Jeong WC, Sajib SZ, Katoch N, Kim HJ, Kwon OI, Woo EJ. Anisotropic conductivity tensor imaging of in vivo canine brain using DT-MREIT. IEEE Trans Med Imaging 2017;36:124-131.

18. Jeong WC, Sajib S, Kim HJ, Kwon OI. Focused current density imaging using internal electrode in magnetic resonance electrical impedance tomography (MREIT). IEEE Trans Biomed Eng 2014;61: 1938-46.

19. Lee JW, Kim D, Yoo S, Lee H, Lee GH, Nam Y. Emerging neural stimulation technologies for bladder dysfunctions. Int Neurourol J 2015;19:3-11.

20. Wei XF, Grill WM. Current density distributions, field distributions and impedance analysis of segmented deep brain stimulation electrodes. J Neural Eng 2005;2:139-47.
21. Lopez-Quintero SV, Datta A, Amaya R, Elwassif M, Bikson M, Tarbell JM. DBS-relevant electric fields increase hydraulic conductivity of in vitro endothelial monolayers. J Neural Eng 2010;7:16005.

22. Kochunov P, Glahn DC, Lancaster J, Thompson PM, Kochunov V, Rogers B, et al. Fractional anisotropy of cerebral white matter and thickness of cortical gray matter across the lifespan. Neuroimage 2011;58:41-9.

23. Sekino M, Ohsaki H, Yamaguchi-Sekino S, Iriguchi N, Ueno S. Low-frequency conductivity tensor of rat brain tissues inferred from diffusion MRI. Bioelectromagnetics 2009;30:489-99.

24. Miranda PC, Mekonnen A, Salvador R, Ruffini G. The electric field in the cortex during transcranial current stimulation. Neuroimage 2013;70:48-58. 